\title{
Performance of Basic Strategies for following Gradients in Two Dimensions
}

\author{
David B. Dusenbery*
}

School of Biology, Georgia Tech, Atlanta, GA 30332-0230, U.S.A.

(Received on 11 November 1999, Accepted in revised form on 28 October 2000)

\begin{abstract}
Computer models for following stimulus gradients in two-dimensional space were evaluated to determine the relative advantages of different strategies and to identify the issues that must be addressed in making such a comparison. The simulations were implemented with emphasis on making them as general and free of specific assumptions as possible. Performance was defined as progress along the gradient divided by the cost of the movements and time taken. Plausible values of costs were taken from data on animal energetics. The models also included various kinds of noise that limit performance. These included unintended variations and biases in motor outputs as well as sensory inputs. An initial guess at appropriate noise levels led to performance worse than that observed in experiments with leukocytes. Reduced noise levels gave good agreement. Under these, more appropriate, conditions, peak performance for the various models varied from 24 to $99 \%$ of the maximum possible. The threshold gradient required to provide performance equal to $1 \%$ of the maximum possible varied from 800 to 5000 searcher diameters per gradient decay length. Some models performed well only over a narrow range of gradients. There was no indication of a tradeoff between sensitivity to shallow gradients and high performance in steep gradients. The model of tropotaxis (simultaneous, spatial comparison) with movement in any direction was superior in having the lowest threshold, the highest maximum performance, requiring the fewest parameters to fit, and performed well over the widest range of gradients. This result suggests that amoeboid cells and echinoderms might be particularly well suited to following gradients. The modeling demonstrates the need to obtain quantitative estimates for a number of parameters (relating costs and noise levels) for a more rigorous understanding of the relative advantages of these different strategies.
\end{abstract}

(C) 2001 Academic Press

\section{Introduction}

Students of the behavior of "simple" animals have long recognized several classes of behavior that are employed to solve the simple problem of following a stimulus gradient to higher or lower intensities (Burr, 1984; Carlile, 1980; Dunn, 1990; Dusenbery, 1992, pp. 413-416; Ewer \& Bursell, 1950; Fraenkel \& Gunn, 1961; Gunn et al., 1937; Jander, 1975; Keller et al., 1977; Schöne, 1984, pp. 35, 39-41). Quantitative analysis of some of

\footnotetext{
*E-mail: david.dusenbery@biology.gatech.edu
}

the "strategies" have been made (Benhamou \& Bovet, 1989; Bornbusch, 1984; Bornbusch \& Conner, 1986; DeLisi, 1982; Doucet \& Drost, 1985; Dusenbery, 1989a, b; Ferrée \& Lockery, 1999; Lauffenburger, 1982; Pierce-Shimomura et al., 1999; Rohlf \& Davenport, 1969; Schnitzer et al., 1990; Tranquillo, 1990; Van Houten \& Van Houten, 1982) to understand the behavior of particular organisms, but there has been little quantitative comparison of the relative effectiveness of the various strategies. Such information is essential to a full understanding of why different 
strategies have evolved in different lineages, why different strategies are employed by the same individual in different circumstances, and may help the engineer in the design of autonomous vehicles. In addition to providing an initial comparison, a major goal of the analysis presented here is to identify issues that must be considered in comparing different strategies and to develop a common language for describing the various strategies.

Since the nonlinear and history-dependent behavior of organisms defies rigorous mathematical analysis, computer simulation was employed. This leads to explicit description of the strategies and conditions and allows thousands of replications to be performed in order to obtain welldefined descriptions of performance, even though there is significant variation in the performance of a particular strategy in a particular condition.

The basic types of strategies have been reviewed (Burr, 1984; Dusenbery, 1992, pp. 413-431; Schöne, 1984, pp. 34-41, 57-69), and the three most important are considered here: klinokinesis, klinotaxis, and tropotaxis. Klinokinesis includes strategies in which the adequate stimulus is a temporal change in intensity and the response is a change in the rate or probability of change of direction but the direction chosen is unrelated to the direction of the gradient. It can often be described as a biased random walk, in which the stimulus modulates the lengths of the steps. The basic strategy can also be described as "if conditions are improving, keep on in the same direction, otherwise try a new direction". The well-studied chemotaxis of bacteria employs this strategy (Berg, 1985) and ciliates probably do (Van Houten, 1978). Klinotaxis includes strategies in which the adequate stimulus is also a temporal change in intensity but the response includes changes of direction that are biased in the direction of the gradient. A single receptor can be moved in some defined pattern that samples the gradient at different locations to obtain the necessary information. This strategy is thought to be employed by nematodes (Burr, 1984; Dusenbery, 1987; Ward, 1973) and swimming algae (Foster \& Smyth, 1980). Tropotaxis includes strategies in which more than one receptor are used to simultaneously sample the gradient at different locations, providing a spatial comparison. With this information a response in the appropriate direction along the gradient is possible. This strategy is part of the behavior of amoeboid cells (Segall, 1990; Tranquillo, 1990) and probably echinoderms (Moore \& Lepper, 1997).

Each strategy type was represented by several models that were defined by three equations (specifying movement distance, direction, and rotation) with several adjustable parameters. Each model was formulated with the idea of keeping it as simple as possible, while retaining the essential features of the strategy. A model with specific

TABle 1

Concepts used

\begin{tabular}{ll}
\hline Concept & \\
\hline Strategy type & Klinokinesis, Klinotaxis, or Tropotaxis \\
Strategy & A strategy type $+\#$ receptors + type of movement $(1,2,3,4$, or 5$)$ \\
Model & $\begin{array}{l}\text { A strategy }+ \text { indicator of whether there is a continuous }(\mathrm{n}) \text { or discontinuous (t) relationship between } \\
\text { sensory input and motor output }(1 \mathrm{n}, 1 \mathrm{t}, 2 \mathrm{n}, 2 \mathrm{t} \text {, etc. })\end{array}$ \\
Implementation & $\begin{array}{l}\text { A specific model }+ \text { specific values for all the parameters specified by the model }\left(\alpha_{i n c}, \alpha_{d e c}, G_{S},\right. \\
\left.V_{S}, T_{S}, P_{S}\right)\end{array}$ \\
Searcher & $\begin{array}{l}\text { An individual organism or robot that includes a specific implementation }+ \text { bias factors }\left(B_{T}, B_{R}\right)+\text { a } \\
\text { starting orientation }\left(\alpha_{0}\right)\end{array}$ \\
Environment & $\begin{array}{l}\text { All other parameters that act as constraints on performance (size, noise, costs, initial memory values, } \\
\text { length of simulation run, radius of search area) }\end{array}$ \\
\hline
\end{tabular}


values for these parameters is called an implementation of the model (Table 1). Quantitative values for the parameters that gave rise to good performance within the constraints of the model were identified by a specialized optimization procedure and examination of contour plots of performance over arrays in which a pair of parameters was varied. The latter provided insight into the volume of parameter space with good performance and the ease with which good performance can be found for a given model.

A particular set of rounded parameter values that provided performance close to the best observed was established for each model. These were taken as standard values, and the whole set defined as a standard implementation of the model. The sensitivity of each model to parameter values was characterized by determining, for each parameter, the range of values over which performance was within 0.5 of performance using the standard implementation.

Performance is here defined as progress (distance moved in the direction of the gradient) per unit cost: performance $=$ progress $/$ cost. An attempt is made to keep the simulations as general as possible, but the comparisons require that specific values be assigned to the various kinds of costs of movement. Although a lack of information makes this assignment rather arbitrary, any comparison involves at least implicit assignment of such values, and it is better to make this explicit. The assignment was made using values that are at least plausible for animals and internally consistent.

\section{The Environment}

\section{GRADIENT}

An exponential gradient was chosen because it provides a uniform stimulus situation to organisms with the common characteristic of responding in a constant way to a given relative change and commonly occurs. Thus, intensity at a location $x, y$ along the gradient parallel to the $x$-axis was defined as: $I(x, y)=\exp (x / d)$, where $x$ and $y$ are the \pm distances from a central point along orthogonal axes, and $d$ is the decay length of the gradient, which is the distance over which intensity changes by a factor of $e$ and is also the reciprocal of the relative gradient. In principle, such an exponential gradient occurs where a light beam is absorbed by uniform material or a chemical concentration is kept constant in one layer by rapid mixing and the chemical is consumed in an adjacent layer at a rate proportional to its local concentration without mixing. (In open water, such smooth gradients occur only at small size scales.)

NOISE

Noise of various kinds will degrade performance, and some strategies may be more susceptible than others. Noise varying more or less randomly in time is commonly considered with regard to signals. However, inevitable, unintended variations in motor outputs also degrade performance and may be considered a type of noise (Dusenbery, 1989a, b). I will ignore noise effects in memory, since it is under more control of the searcher than either the inputs or outputs, and I assume that memory will be designed to not add significant noise. In principle, memory can be made with any degree of reliability at low cost (Dusenbery, 1992, pp. 43, 44).

In addition to variation between time intervals, there is inevitable variation between the components assembled in a given searcher. These variations produce biases in the performance of a given individual, and these effects have been termed "developmental noise" (Dusenbery, 1989a, 1992, p. 418). These considerations lead to the identification of four types of noise, as displayed in Table 2.

Intensity noise is temporal variation in the relation between the input and output of a receptor. It is the most familiar type of noise. It was emulated by multiplying the intensity at the receptor by a noise factor $\left(F_{I}\right)$, newly sampled at each time step.

Motor noise is unintended variations in the movement of the searcher. It was emulated by multiplying the intended rotation and distance of translation at each step by noise factors $\left(F_{R}, F_{D}\right)$, newly sampled at each time step.

Receptor bias results from unintended differences in the receptors and results in consistent bias in the outputs of different receptors within an individual searcher. It was modeled by 
TABle 2

Types of noise

\begin{tabular}{lcc}
\hline & \multicolumn{2}{c}{ Effects are on } \\
\cline { 2 - 3 } Variation is between & Receptor inputs & Motor outputs \\
\hline Time intervals & Intensity noise $\left(N_{I}, N_{A}\right)$ & Motor noise $\left(N_{T}, N_{R}, N_{D}\right)$ \\
Individual receptors or searchers & Receptor bias $\left(N_{R B}\right)$ & Motor bias $\left(N_{T B}\right)$ \\
\hline
\end{tabular}

The parentheses enclose symbols for parameters employed in the models as defined in Table 3.

TABLE 3

Standard values of environmental parameters

\begin{tabular}{lccc}
\hline Parameter & Symbols & Set 1 & Set 2 \\
\hline Intensity noise & $F_{I} \leftarrow N_{I}=$ & 0.1 & 0.01 \\
Receptor bias & $B_{R} \leftarrow N_{R B}=$ & 0.1 & 0.01 \\
Adaptation noise & $F_{A} \leftarrow N_{A}=$ & 0.1 & 0.01 \\
Distance noise & $F_{D} \leftarrow N_{D}=$ & 0.1 & 0.01 \\
Rotation noise & $F_{R} \leftarrow N_{R}=$ & 0.1 & 0.01 \\
Turning bias & $B_{T} \leftarrow N_{T B}=$ & 0.01 & 0.01 \\
Turning noise & $N_{T}=$ & 0.1 & 0.01 \\
Cost of translation & $C_{\text {Trans }}$ & 0.06 & 0.009 \\
Cost of rotation & $C_{\text {Rot }}$ & 4 & 0.028 \\
Cost of time & $C_{\text {Time }}$ & 0.4 & 0.001 \\
Speed limit & $V_{\text {Max }}$ & 20 & 1 \\
& $r$ & 10 & 0.5 \\
Searcher radius & $r$ & $300 V_{S}$ & 100 \\
Arena radius & & 300 & 300 \\
Time limit & & 100 & 100 \\
Gradient for optimization & & 200 & 100 \\
Threshold gradient $(S / N=1)$ & & 10.5 & 100 \\
Threshold gradient/searcher diameter & & 100 \\
Maximum performance & & & \\
\hline
\end{tabular}

In the last three rows of the table general consequences of the parameters are given.

assigning to each receptor an independent noise factor that was constant throughout a $\operatorname{run}\left(B_{R}\right)$.

Motor bias results from unintended differences in the motor components and results in consistent bias in the locomotion of individual searchers. It was modeled by assigning to each searcher an independent noise factor that was added to the turn at each time step $\left(B_{T}\right)$. The factor for each individual was fixed during a run.

These noise factors were drawn from a lognormal distribution with median one. Using $G(m, \sigma)$ to represent a randomly chosen value from a Gaussian distribution of mean $m$ and standard deviation $\sigma$, the noise factors are of the form
$F_{X}=\exp \left(G\left(0, N_{X}\right)\right)$. The $F$ factors are resampled at each time step.

Similar factors $(B)$ represent the effects of biases due to developmental or construction noise, $B_{X}=\exp \left(G\left(0, N_{X B}\right)\right)$, but they differ in being selected for each searcher at the start of a run and remain constant during a run. For the results reported here, $N_{X}$ and $N_{X B}=0.1$ or 0.01 . With these values for noise, $68 \%$ of factors are between 0.9 and 1.1 times the nominal parameter value for the larger noise value and between 0.99 and 1.01 for the smaller value. The larger value was originally thought to be a good general representation of the performance of most real-world components. The values of the noise parameters employed in these studies are presented in Table 3. 


\section{SIGNAL-TO-NOISE RATIO}

The signal-to-noise ratio $(S / N)$ available to a searcher in these conditions can be calculated as follows. The best possible signal (for klinotaxis or tropotaxis) is the difference in intensity at opposite sides of the searcher in line with the gradient. If the searcher fits in a circle of radius $r$, the best possible signal is $S=\mid I(x-r)-$ $I(x+r)|=| \mathrm{e}^{(x-r) / d}-\mathrm{e}^{(x+r) / d}\left|=\mathrm{e}^{x / d}\right| \mathrm{e}^{-r / d}-\mathrm{e}^{+r / d} \mid$. The noise is taken as the standard deviation of the underlying Gaussian distribution of intensity noise, $\quad N=I(x) N_{I}=\mathrm{e}^{x / d} N_{I}$. Thus, we get $S / N=\left|\mathrm{e}^{-r / d}-\mathrm{e}^{+r / d}\right| / N_{I}$. Under conditions of interest, $r \ll d$, and the exponentials can be approximated with the series representation using only terms up through the linear term. This gives $S / N=2 r / d N_{I}$.

A signal-to-noise ratio of unity is often taken as an indication of the threshold of significant response, and we see that $N_{I}$ equals the number of searcher diameters per gradient decay length giving a signal-to-noise ratio of unity. For parameter set $1, N_{I}=0.1$, and $S / N=1$ where the gradient decay length is 200 spatial units or ten searcher diameters. For parameter set 2, $N_{I}=0.01$, and $S / N=1$ where the gradient decay length is 100 searcher diameters.

\section{The Searchers}

\section{THE GENERAL SEARCHER MODEL}

Stimuli in the environment of the searcher impinge on a set of $n$ receptors $\left(R_{1}, R_{2}, \ldots, R_{n}\right)$ distinguished only by their spatial location and receptor bias factor. These receptors are arrayed around the searcher at a radius $r$, and each is associated with a direction $\left(\theta_{1}, \theta_{2}, \ldots, \theta_{n}\right)$ from the forward axis of the searcher. Each receptor outputs an analog signal represented as a real number.

The receptors send signals to one or more members of a set of $m$ analog memory elements $\left(M_{1}, M_{2}, \ldots, M_{m}\right)$, also represented by a real number.

The memories are combined to control the intended behavioral output of the searcher: the turning rate (change in orientation of the axis of the searcher),

$$
T(t)=\mathbf{B}_{T}\left(M_{1}(t), M_{2}(t), \ldots, M_{m}(t)\right),
$$

the direction of locomotion with respect to the axis of the searcher,

$$
D(t)=\mathbf{B}_{D}\left(M_{1}(t), M_{2}(t), \ldots, M_{m}(t)\right)
$$

and the speed of locomotion (distance translated per time step in the direction defined above),

$$
V(t)=\mathbf{B}_{V}\left(M_{1}(t), M_{2}(t), \ldots, M_{m}(t)\right)
$$

This allows translation to be confined to straight ahead, if $D(t)=0$ is always true, or to be in another direction as occurs in amoeboid cells, echinoderms, and crabs.

Specific strategies are defined by the three B functions and the $n \times m$ potential signalprocessing connections between receptors and memories. In the work reported here, there is a one-to-one relationship between memories and receptors, and $M_{i}(t+\Delta t)=P_{i}\left(M_{i}(t), R_{i}(t+\Delta t)\right)$. (Given this restraint, the subscript is unnecessary and will be dropped from here on, with the understanding that each memory is influenced by one and only one receptor.) The signal-processing functions $P$ include the effects of sensory adaptation as well as connections between memory elements and receptors. In more complicated models, the $P$ 's might include inputs from more than one receptor and perhaps inputs from other memories.

\section{Sensory Adaptation}

Sensory adaptation is a nearly universal feature of biological sensory systems (Dusenbery, 1992, pp. 95-105; Withers, 1992, pp. 261-263), and I use a model that provides the basic features of sensory adaptation:

$$
\begin{aligned}
M(t+\Delta t)= & M(t)+\alpha F_{A}(R(t+\Delta t) \\
& -M(t)) \Delta t,
\end{aligned}
$$

where $F_{A}=\exp \left(G\left(0, N_{A}\right)\right)$ is a noise factor with a new value at each time step, and $\alpha(0<\alpha<1 / \Delta t)$ is a parameter controlling the rate of adaptation. $M$ changes toward the present receptor value with more or less time lag and dependence on past stimulation levels. When 
$\alpha$ has its maximum value $(\alpha=1 / \Delta t)$, $M(t+\Delta t)=R(t+\Delta t)$ on average, and there is effectively no adaptation since the memory element follows the receptor output as closely as noise permits. The resulting behavior could be modeled by assuming that the receptor output directly controls behavior and there is no need for invoking a memory element. If $\alpha=0$, the memory element retains its initial value, and input from receptors has no effect. With intermediate values, the history of stimulation has an effect, with smaller values extending the relevant history back further in time. With a step change in intensity, $M$ makes an exponential change from the old to the new value, and $\alpha$ determines the rate of this change (Dusenbery, 1989a).

It has been observed (Dusenbery, 1989b) that in a wide variety of organisms adaptation is asymmetrical with regard to increasing and decreasing intensities, and modeling indicates that such asymmetry can increase the efficiency with which a gradient can be followed. Consequently, the model provided for independent adaptation rates for increasing and decreasing intensities:

if $R(t+\Delta t)>M(t)$

$$
\begin{aligned}
& \text { then } M(t+\Delta t)=M(t)+\alpha_{i n c} F_{A}(R(t+\Delta t) \\
& \quad-M(t)) \Delta t
\end{aligned}
$$$$
\text { else } M(t+\Delta t)=M(t)+\alpha_{d e c} F_{A}(R(t+\Delta t)
$$$$
-M(t)) \Delta t
$$

\section{Intensity Contrast}

For several types of behavior a subjective intensity factor $\left(I_{C}\right)$ is calculated as a contrast between recent stimulus intensities $\left(M_{1}\right)$ and intensities experienced in the more distant past $\left(M_{2}\right)$ :

$$
I_{C}=\left\{\begin{array}{l}
1 \quad \text { if } M_{1}=M_{2}-0 \\
\mathrm{e}^{G_{S}\left(M_{1}-M_{2}\right) /\left(M_{1}+M_{2}\right)}
\end{array}\right.
$$

where $G_{S}$ is a model parameter controlling sensitivity and representing gain in the system. $I_{C}$ has the property that it is unity whenever $M_{1}=M_{2}$, irrespective of the value of gain, and takes larger values when intensity is increasing and smaller values when intensity is decreasing. Note that changing the sign of the gain causes $I_{C}$ to take the reciprocal value, reversing the direction of change: $I_{C}\left(G_{S}, M_{1}, M_{2}\right)=1 / I_{C}\left(-G_{S}, M_{1}, M_{2}\right)=$ $I_{C}\left(-G_{S}, M_{2}, M_{1}\right)$.

\section{Input-Output Relations}

Each of the types of behavioral strategies is represented by two specific models. One model ( $t$ ) contains a threshold and has a discontinuous relation between receptor input and motor output. The other model (n) does not have a threshold and has a continuous relation between receptor input and motor output.

\section{SPECIFIC SEARCHER MODELS}

\section{A Biased Random Walk Employing Sequential Sampling and Indirect Response (Klinokinesis)}

This strategy requires one receptor spread out over the surface and providing a single average output equal to a receptor at the center of the searcher. Two memories are described, but one memory merely passes on the current output of the receptor $\left(\alpha_{i n c, 1}=\alpha_{d e c, 1}=1\right)$, so only one memory and one receptor are essential. $M_{1}$ follows the sensory input as faithfully as noise permits, while $M_{2}$ lags behind, carries information about past experience, and tends to the input but swings above and below it with increasing or decreasing trends. This causes $I_{C}(t)$ to swing above and below 1 . The models move forward, $D(t)=0$, continuously at a constant speed, $V(t)=V_{S}$. (The subscript $S$ indicates that the parameter is constant for any particular searcher.)

The controlling relation of the threshold model (labeled 1t) was

$$
\begin{aligned}
& \text { if } I_{C}(t) R_{\text {Random }}(0,1)<P_{S} \\
& \text { then } T(t)=R_{\text {Random }}\left(-T_{S}, T_{S}\right) \\
& \text { else } T(t)=0,
\end{aligned}
$$

where $R_{\text {Random }}(a, b)$ is a real random number taken from a uniform distribution on $[a, b]$ and 
$P_{S}$ is a constant for each searcher which equals the probability of changing direction under constant stimulation, and $T_{S}$ is a parameter determining the maximum size of the turn.

The controlling relation for the non-threshold model $(\ln )$ was

$$
T(t)=G\left(0, I_{C}(t)\right)
$$

Directions are in units of fractions of a rotation, throughout this paper.

In both models, with appropriate values, the searcher tends to continue moving in a given direction as long as the stimulation is increasing, but if stimulation is decreasing, it is more likely to try a new random direction. $I_{C}$ provides information on the current state of the environment compared with recently experienced states. Note that the organism never has explicit information on the direction of the gradient and whenever it changes direction it is as likely to turn up as down the gradient. This strategy emulates the behavior of free-swimming bacteria (Berg, 1985; Berg \& Tedesco, 1975) and paramecium (Van Houten, 1978).

\section{A Single Receptor with Constant Rotation and Directed Responses (Klinotaxis)}

Klinotaxis is similar to klinokinesis in that it involves one receptor and one or two memories but differs in that the receptor is moved in some pattern and turns are biased in the direction of the gradient. In this strategy (2), the searcher rotates continuously $T(t)=T_{S}$ and translation is always in the direction of the receptor from the center of the searcher $\left(D(t)=\theta_{1}=0\right)$. The stimulus modulates the speed of translation in this direction.

The controlling relation for the threshold model (2t) was

$$
V(t)= \begin{cases}V_{S} & \text { if } I_{C}(t)>1 \\ 0 & \text { if } I_{C}(t) \leqslant 1\end{cases}
$$

The relation for the non-threshold model (2n) was

$$
V(t)=V_{S} I_{C}(t)
$$

This strategy is analogous to the phototactic behavior of many free-swimming algae, which swim in a helical path, with constant rotation. The photoreceptor is directed to the side and scans the environment (Foster \& Smyth, 1980).

\section{A Single Receptor with Oscillation and Directed Responses (Klinotaxis)}

This strategy is similar to the previous strategy (2), except that turning is oscillatory. If the period $P_{S}=0$, then $T(t)=0$, otherwise, for the threshold model (3t),

$$
T(t)=T_{S} \sin \left(2 \pi t / P_{S}\right)
$$

and $V(t)$ is the same as model $2 \mathrm{t}$.

For the non-threshold model (3n)

$$
\begin{aligned}
& T(t)=T_{S} I_{C}(t) \sin \left(2 \pi t / P_{S}\right), \\
& V(t)=V_{S} .
\end{aligned}
$$

These strategies may emulate the behavior of nematodes, which swing their heads back and forth across their path of forward locomotion (Dusenbery, 1987; Ferrée \& Lockery, 1999; Morse et al., 1998; Ward, 1973).

\section{Spatial, Simultaneous Comparison (Tropotaxis) with a Bilateral Pair of Receptors, Rotation, and Forward Locomotion}

The searcher has two bilateral receptors separated by an angle $P_{S}$. Each sends signals to a separate memory element, in a parallel, symmetrical fashion. The two memories are compared and the searcher turns to head in a more favorable direction. The searcher moves forward $(D(t)=0)$ continuously at a constant speed, $V(t)=V_{S}$.

For the threshold model (4t),

$$
\text { if } \begin{aligned}
M_{i}=M_{j} & \text { then } T(t)=0 \\
& \text { else } T(t)=T_{S} \theta_{i},
\end{aligned}
$$

where $\theta_{i}$ is the angle from the forward axis of the searcher to receptor $i$ and $M_{i}>M_{j}$. 
For the discontinuous model (4n)

$$
T(t)=\left\{\begin{array}{c}
0 \quad \text { if } M_{i}=M_{j}, \\
T_{S}\left(\frac{M_{i}-M_{j}}{\left|M_{i}-M_{j}\right|}\right)\left|\frac{M_{i}-M_{j}}{M_{i}+M_{j} \mid}\right|^{G_{S}},
\end{array}\right.
$$

where $G_{S}$ is a gain factor, constant for each searcher. The first ratio simply provides the sign of the difference and turn, and the second ratio determines the magnitude of the turn.

This strategy emulates familiar behavior and is applicable to arthropods, such as insects and crustaceans.

\section{Spatial, Simultaneous Comparison (Tropotaxis) with many Receptors and Locomotion in any Direction}

This strategy requires more than one receptor, and the receptors send signals to a comparator, in a parallel fashion. Memories are not required, but the implementation assumes that all signals pass through a memory; there is one memory for each receptor. Models with 3-12 receptors were simulated, but initial results indicated continuous improvement in performance with increasing number of receptors, so only models with 3 and 12 receptors were studied in detail. No turning is commanded, $T(t)=0$, in any of these models, although some change of orientation may occur due to rotation bias $\left(B_{T}\right)$ or noise associated with translation $\left(F_{T}\right)$.

In the threshold models $(5 \mathrm{t})$, the searcher simply moves in the direction of the most favorably stimulated receptor $\left(D(t)=\theta_{i}\right.$ where $\left.M_{i}>M_{j \neq i}\right)$ at constant speed, $V(t)=V_{S}$.

In the non-threshold models $(5 n)$, the position of the weighted average of the memories is computed, in analogy with the center of mass, providing a direction $\left(\theta_{m}\right)$ and magnitude $\left(I_{m}\right)$. These values modulate both the direction $\left(D(t)=\theta_{m}\right)$ and speed $\left(V(t)=V_{S} I_{m}^{G_{S}}\right)$ of translation, where $G_{S}$ is a gain parameter, fixed for each searcher.

This strategy is likely to be applicable to amoeboid cells and echinoderms such as sea stars and sand dollars (Moore \& Lepper, 1997).

\section{The Simulations}

\section{COSTS}

In order to compare different strategies, it is necessary to have some measure of the cost of constructing an appropriate searcher and execution of the strategy. I assume that information can be transmitted between locations within the searcher rapidly at low cost, and I ignore such time delays and costs. In contrast to the low cost of spatial transmission, I assume that transmission in the time domain requires relatively costly memory. With these assumptions, functional units of the signal-processing systems that are essential to document are: sensory receptors, memory, and motor outputs.

Initially, I assume that the complexity of the information processing system is best measured through the minimum number of receptors and memory elements required to implement the strategy. Specific costs can later be estimated by assigning specific values to receptors and memory elements.

The cost of motor outputs are relatively idiosyncratic (specific to particular mechanisms and environments), but any comparison of different strategies must (explicitly or implicitly) make some assumptions about these and other costs. The models specify the types of outputs required in terms of abilities to move in more than one direction (as crabs often do) and to rotate in place, in addition to the universal ability to move forward. Thus, the performance of a specific searcher during a run is measured as

$$
\text { Performance }=\frac{\Delta x}{C_{\text {Trans }} L+C_{\text {Rot }} R+C_{\text {Time }} t},
$$

where $\Delta x$ is the distance moved along the gradient, the $C$ 's are the costs of translation, rotation, and time, respectively, $L$ is the total path length traveled, $R$ is the total of rotations, and $t$ is the elapsed time of the run.

For the sake of comparison, it is necessary to assume some specific values for these costs of movement. In an attempt to be as universal as practical, specific values are taken from typical values of energy expenditure in animals. Thus, cost is measured in units of energy $(\mathrm{J})$. As generally assumed, the cost is proportional to the distance moved or time. 
In the first set of runs, the searcher was assumed to be enclosed in a circle of radius 10 units. If we interpret these units as centimeters, a spherical animal would have a volume of 41 and a mass of $4 \mathrm{~kg}$. The time step in the simulation is assumed to be $0.1 \mathrm{~s}$, which is close to the shortest integration times of many stimuli in a variety of animals. (It should be understood that the simulation results could be used with other interpretations of the spatial scale and time step.)

\section{Cost of Translation}

The cost of translation by a variety of mechanisms in air and water has been determined for many animals. For a $4 \mathrm{~kg}$ animal, a cost of $6 \mathrm{~J} \mathrm{~m}^{-1}$ is typical (Peters, 1983, pp. 92, 3), and the value $0.06 \mathrm{~J} \mathrm{~cm}^{-1}$ is taken as the cost of translation per spatial unit in evaluating performance; $C_{\text {Trans }}=0.06$, in the first set of runs.

\section{Cost of Rotation}

No data were found for the cost of rotating in place. Thus, I assumed that the cost of rotation was the same as translation, a distance equal to the distance moved by the circumference of the searcher during the rotation. This accurately represents a searcher with locomotor apparatus (legs or wheels) at the periphery and equal efficiency of forward and backward movements. With a searcher of radius $10 \mathrm{~cm}$, the circumference is $63 \mathrm{~cm}$, so the cost of one complete rotation equals the cost of translating $63 \mathrm{~cm}$, which is about $4 \mathrm{~J}$. The value 4 is then taken as the cost of one full rotation, and other rotations have proportional costs, $C_{\text {Rot }}=4$, in the first set of runs. All directions are measured in units of fractions of a rotation.

\section{Cost of Time}

There is also a cost of time. Taking more time to obtain resources delays reproduction of organisms and other goals of robotic vehicles. For a general estimate of this cost, I use the specific metabolic rate of typical animals $\left(\mathrm{J} \mathrm{s}^{-1}\right.$ $\left.\mathrm{kg}^{-1}=\mathrm{W} \mathrm{kg}^{-1}\right)$. This is the minimum rate at which energy is consumed and must be replaced by obtaining food or fuel. I use the value
$1 \mathrm{~W} \mathrm{~kg}^{-1}$, which is intermediate between warmand cold-blooded animals of all sizes (Peters, 1983, p. 32, Fig. 3.2). For the $4 \mathrm{~kg}$ animal assumed in the first set of runs, energy is consumed at a rate of $4 \mathrm{~W}$ or $4 \mathrm{~J} \mathrm{~s}^{-1}$. Since the time step is interpreted as $0.1 \mathrm{~s}$, the value 0.4 is then taken as the cost of time per step, $C_{\text {Time }}=0.4$, in the first set of runs.

In the second set of runs, environmental parameter values were chosen to provide more convenient reference values. Searcher diameter was set equal to the spatial unit. The costs $\left(C_{\text {Trans }}=0.009, C_{\text {Time }}=0.001\right)$ were chosen to provide a maximum performance of 100 . These costs are consistent with the previous time unit of $0.1 \mathrm{~s}$, a spatial unit of $1 \mathrm{~cm}$, and a searcher volume of about $1 \mathrm{~cm}^{3}$, with a cost unit of $0.1 \mathrm{~J}$.

The relative costs of translation and time are reversed between environmental parameter sets 1 and 2, because of the difference in assumed size. The cost of time is proportional to volume, while the cost of translation varies less with volume.

\section{OTHER CONSTRAINTS}

\section{Speed Limit}

In the course of carrying out the simulations, it was discovered that another important constraint is that there is a maximum possible speed. Considering reported speeds of swimming organisms of all kinds, indicates that 10 lengths s ${ }^{-1}$ is typical across all sizes (Dusenbery, 1996, p. 45; Mann \& Lazier, 1991, p. 16). Few swimming organisms differ by more than an order of magnitude from this value. Equating length with diameter, a diameter of $20 \mathrm{~cm}$, would typically have a speed of $200 \mathrm{~cm} \mathrm{~s}^{-1}$. This is equivalent to 20 spatial units per time step, and $V_{M a x}=20$ is applied to all simulations in the first set of runs. For the second set, similar considerations lead to a $V_{\text {Max }}=1$. (These limits may be optimistic, in that organisms that move across surfaces usually move more slowly than those that swim free of contact with surfaces.)

\section{Maximum Performance}

With these values specified, there is a clearly defined maximum possible performance. This 
occurs when the searcher moves straight along the axis of the gradient, without rotating, at maximum speed. Then, the maximum possible performance is equal to $\left(C_{\text {Trans }}+\left(C_{\text {Time }} / V_{\text {Max }}\right)\right)^{-1}$, with values shown in Table 3 .

\section{PERFORMANCE EVALUATION}

Performance was evaluated by computer simulation. Each searcher was started at a central location facing in a randomly chosen direction. All parameter values for a given searcher were fixed except for receptor inputs, memory, and movement outputs. Initial memory values were 0 . Receptor values $\left(R_{r}(t)\right)$ were taken from receptor position $\left(x_{r}\right)$ in the gradient with noise factors: $R_{r}(t)=B_{R} F_{I} I\left(x_{r}(t)\right)$, where the noise factors $B_{R}$ are fixed for each receptor and $F_{I}$ is a new sample at each time step. Memory was modified according to adaptation rates [eqn (5)], and movement was made according to the relations described in Section 3.2.

The noise effects on behavior were implemented as follows. For turning:

$$
\begin{aligned}
\alpha(t+1)= & \alpha(t)+F_{R} T(t+1) \\
& +G\left(B_{T},\left(N_{T} D(t+1) / r\right),\right.
\end{aligned}
$$

where $\alpha(t)$ is the orientation of the searcher axis with respect to the environment at time $t, T(t)$ is the turn specified by the model at time $t, F_{R}$ is a new sample of the noise factor at each time step, $B_{T}$ is the turning bias of the searcher as a result of developmental or construction noise with a value taken from $G\left(0,\left(N_{T B} V_{S} / r\right)\right)$ at the beginning of a run $\left(N_{T B}\right.$ is the parameter controlling the magnitude of turning bias), $N_{T}$ is the turning noise parameter, and $r$ is the radius of the searcher. Thus, the searcher tends to wander in unintended directions by $N_{T}$ rotations when it translates a distance equal to its radius and has a bias to turn $B_{T}$ rotations in each time step.

For position,

$$
\begin{aligned}
& x(t+1)=x(t)+V(t+1) F_{D} \sin (2 \pi D(t+1)), \\
& y(t+1)=y(t)+V(t+1) F_{D} \cos (2 \pi D(t+1)),
\end{aligned}
$$

where $x(t)$ and $y(t)$ specify the position of the searcher at time $t$, and $F_{D}$ is the same value of the distance noise factor for both $x$ and $y$, but newly sampled at each time step.

This process was repeated, step by step for a maximum number of time steps (300) or until the searcher moved beyond the arena radius (Table 3) in any direction. This circular spatial limit avoided any bias in where a given search was terminated.

After all searchers had reached the arena limit or the time limit expired, progress along the gradient, total distance translated, total rotations made, time duration of the search, and performance (progress/cost) were determined for each searcher. Then the distribution of performance values was characterized for the whole population of 10000 searchers in terms of median, mean, standard deviation, skew, and kurtosis. The latter two parameters revealed that the distributions were often far from normal, and the median was used as the best measure of performance of each implementation.

\section{Results}

At the outset, experience in laboratory measurements suggested that appropriate noise standard deviations might generally be about 0.1 , and some studies (not shown) were executed with all noise standard deviations $(N)$ set to this value. Then it was realized that this value for the turning bias factor $\left(N_{T B}=0.1\right)$ leads to movement in quite small circular paths (on the order of one circle in a distance of 10 radii). Subsequently, this factor was set ten-fold lower. Using this set of noise values, and the set of costs derived from assuming the searcher was like a typical animal of radius $10 \mathrm{~cm}$, defined the values of the environmental parameters for the first set of runs, as shown in Table 3.

After some initial exploration, a gradient with a decay length of 100 spatial units or five searcher diameters was identified as being the shallowest gradient that provided useful performance for all 12 strategies. Optimization runs and exploration of two-dimensional arrays of values led to the standard values of the model parameters for each model as shown in Table 4. In all cases, adaptation rates of 1 for the memory with the most 
TABLE 4

Parameter set 1

\begin{tabular}{|c|c|c|c|c|c|c|c|c|c|c|c|c|}
\hline \multirow{2}{*}{\multicolumn{4}{|c|}{ Strategy description }} & \multicolumn{8}{|c|}{ Parameter values that provide good performance } & \multirow[b]{3}{*}{ Performance } \\
\hline & & & & \multicolumn{4}{|c|}{ Adaptation rates } & \multirow[b]{2}{*}{$\begin{array}{c}\text { Speed } \\
\left(V_{S}\right)\end{array}$} & \multirow[b]{2}{*}{$\begin{array}{l}\text { Turn } \\
\left(T_{S}\right)\end{array}$} & \multirow[b]{2}{*}{$\begin{array}{c}\text { Gain } \\
\left(G_{S}\right)\end{array}$} & \multirow[b]{2}{*}{$P_{S}$} & \\
\hline $\begin{array}{l}\text { Strategy } \\
\text { type }\end{array}$ & Model & $\begin{array}{l}\text { Number } \\
\text { receptors }\end{array}$ & $\begin{array}{l}\text { Movement } \\
\text { controlled }\end{array}$ & $\alpha_{i n c, 1}$ & $\alpha_{d e c, 1}$ & $\alpha_{i n c, 2}$ & $\alpha_{d e c, 2}$ & & & & & \\
\hline Klinokinesis & $1 n$ & $(1)$ & Run length & 1 & 1 & 0.1 & 1 & 5 & - & 100 & - & 1.0 \\
\hline Klinokinesis & $1 t$ & (1) & Run length & 1 & 1 & 0.1 & 0.5 & 5 & 0.5 & 100 & $0.1^{*}$ & 2.2 \\
\hline Klinotaxis & $2 n$ & 1 & Speed & 1 & 1 & 0.7 & 0.03 & 5 & 0.04 & 60 & - & 2.1 \\
\hline Klinotaxis & $2 t$ & 1 & Speed & 1 & 1 & 0.7 & 0.1 & 14 & 0.05 & - & - & 2.2 \\
\hline Klinotaxis & $3 n$ & 1 & Rotation & 1 & 1 & 0.09 & 1 & 5 & 0.4 & -40 & $1.7 * *$ & 2.9 \\
\hline Klinotaxis & $3 t$ & 1 & Speed & 1 & 1 & 0.8 & 0.1 & 200 & 0.01 & - & $0 * *$ & 2.7 \\
\hline Tropotaxis & $4 n$ & 2 & Rotation & 1 & 1 & 1 & 1 & 5 & 0.3 & 0.8 & $0.5^{* * *}$ & 3.5 \\
\hline Tropotaxis & $4 t$ & 2 & Rotation & 1 & 1 & 1 & 1 & 5 & 0.2 & - & $0.5^{* * *}$ & 3.0 \\
\hline Tropotaxis & $5 n 3$ & 3 & Direction & 1 & 1 & 1 & 1 & 20 & - & 0.1 & - & 5.8 \\
\hline Tropotaxis & $5 t 3$ & 3 & Direction & 1 & 1 & 1 & 1 & 20 & - & - & - & 4.9 \\
\hline Tropotaxis & $5 n 12$ & 12 & Direction & 1 & 1 & 1 & 1 & 20 & - & 0.1 & - & 9.5 \\
\hline Tropotaxis & $5 t 12$ & 12 & Direction & 1 & 1 & 1 & 1 & 20 & - & - & - & 6.3 \\
\hline
\end{tabular}

Constraints: noise $=0.1$, except TurningBiasFactor $=0.01$; costs $=0.06,4,0.4$; searcher radius $=10$. Performance measured at a gradient decay length of 100 units or ten searcher diameters. Maximum possible performance is 12.5 . Adaptation rates limited to $[0,1]$.

${ }^{*} P_{\mathrm{S}}$ is the probability of changing direction.

${ }^{*} * P_{\mathrm{S}}$ is the period of oscillation.

${ }_{* * *} P_{\mathrm{S}}$ is the separation of receptors. 


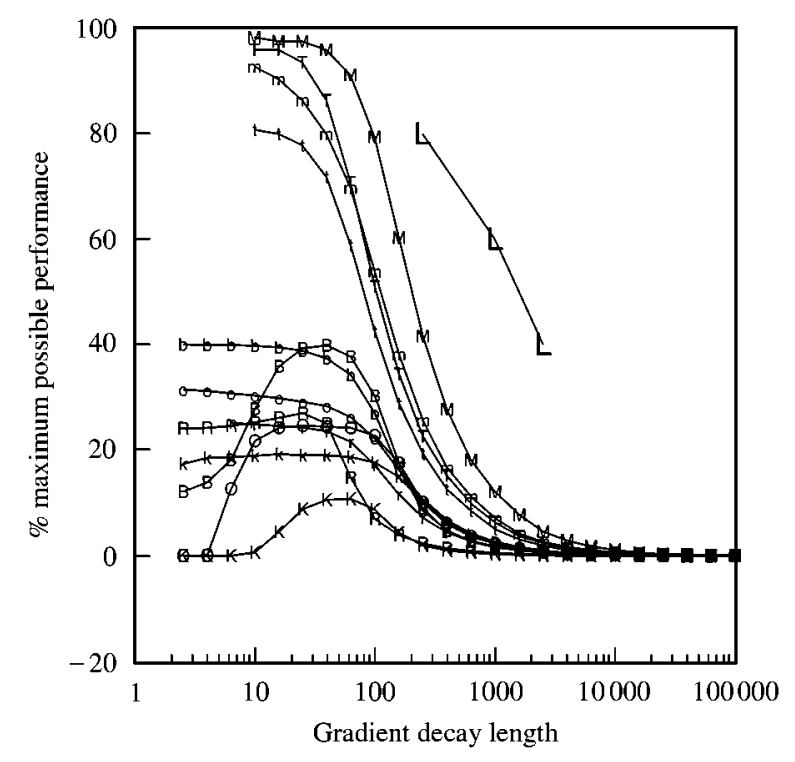

FIG. 1. Performance of strategies under parameter set 1 and data for leukocyte chemotaxis. Gradient steepness declines to the right. The signal-to-noise ratio for searchers is unity at a gradient decay length of 200 or ten searcher diameters. Leukocyte data "L" is from Zigmond (1977). All other symbols represent the median performance of 10000 simulated searchers: $1 \mathrm{n},-\mathrm{K}-; 1 \mathrm{t},-\mathrm{k}-; 2 \mathrm{n},-\mathrm{R}-; 2 \mathrm{t}$, $-\mathrm{r}-; 3 \mathrm{n},-\mathrm{O}-; 3 \mathrm{t},-\mathrm{O}-; 4 \mathrm{n},-\mathrm{B}-; 4 \mathrm{t},-\mathrm{b}-; 5 \mathrm{n} 3 ;-\mathrm{T}-$; $5 \mathrm{t} 3, \stackrel{\mathrm{t}-}{-} 5 \mathrm{n} 12,-\mathrm{M}-; 5 \mathrm{t} 12,-\mathrm{m}-;$ leukocytes, $-\mathrm{L}-$

recent information worked well. With this value the memory simply tracks the output of the receptor, and there would be no need to include a separate memory element. In contrast, for strategies with only one receptor, it was necessary to have a smaller adaptation rate for the second memory, and as previously observed (Dusenbery, $1989 \mathrm{~b})$ there is an advantage to having different rates for increasing and decreasing intensities. The distance moved per step ranged from $1 / 4$ maximum speed up to the maximum speed. Rotation per step was surprisingly small for rotating klinotaxis (strategy type 2), but it was confirmed that these small values were superior to 0 . However, strategy $3 \mathrm{t}$ worked well with no commanded rotation; apparently, rotations caused by noise were sufficient. Gain was very important in strategies with continuous relations between sensory input and motor output.

The results of testing the various models with the standard values of set 1 in a range of gradients are presented in Fig. 1. As gradients became more shallow the performance of all strategies approached zero. At the gradient decay length where $S / N=1$ (200 or 10 searcher diameters), performance varied over $3.2-52 \%$ of the maximum possible. Defining threshold as $1 \%$ of the maximum possible performance, the threshold for all strategies fell in the gradient decay length range of 400-10000 spatial units or $20-500$ searcher diameters. Peak performance for each model varied from 11 to $98 \%$ of maximum possible performance. Only tropotaxis (strategy 5) models attained performance above $40 \%$ of the maximum possible, and all tropotaxis models attained at least $80 \%$ of maximum for sufficiently steep gradients. The two klinokinesis models had the lowest maximum performance-below $20 \%$. Most remarkably, the performance of some models declined as the gradients became very steep. These were all models with continuous relations between input and output. Apparently, in these models, the fixed parameter values adapt the searcher to only a narrow range of gradients.

Figure 1 also includes experimental data from leukocytes (Zigmond, 1977), the only data I found for organisms moving on a substrate in a defined gradient. Remarkably, the leukocytes responded well in gradients an order of magnitude shallower relative to their size than any of the models. This observation led to the conclusion that the noise levels assumed in parameter set 1 were too high. And so the analysis was repeated with all noise standard deviations equal to 0.01 . At the same time, the cost parameters were changed to make the maximum possible performance equal to 100 and to be more consistent with a smaller size scale, relevant to the leukocyte data (Table 3, set 2).

Since noise levels and relative costs had changed, the models were all optimized again, this time at gradient decay lengths of 100 searcher diameters, and the standard parameter values for model parameters in set 2 are presented in Table 5. The results of testing these implementations in a range of gradients are presented in Fig. 2. At the gradient decay length where $S / N=1$ (100 searcher diameters), performance varied over $18-52 \%$ of the maximum possible. The $1 \%$ of maximum possible performance threshold fell in the gradient decay range of 800-5000 searcher diameters, i.e. gradients about an order of magnitude shallower than set 1 . And the 
TABLE 5

Parameter set 2

Parameter values that provide good performance

\begin{tabular}{|c|c|c|c|c|c|c|c|c|c|c|c|c|}
\hline \multicolumn{4}{|c|}{ Strategy description } & \multicolumn{4}{|c|}{ Adaptation rates } & \multirow[b]{2}{*}{$\begin{array}{c}\text { Speed } \\
\left(V_{S}\right)\end{array}$} & \multirow[b]{2}{*}{$\begin{array}{l}\text { Turn } \\
\left(T_{S}\right)\end{array}$} & \multirow[b]{2}{*}{$\begin{array}{c}\text { Gain } \\
\left(G_{S}\right)\end{array}$} & \multirow[b]{2}{*}{$P_{S}$} & \multirow[b]{2}{*}{ Performance } \\
\hline $\begin{array}{l}\text { Strategy } \\
\text { type }\end{array}$ & Model & $\begin{array}{l}\text { Number } \\
\text { receptors }\end{array}$ & $\begin{array}{l}\text { Movement } \\
\text { controlled }\end{array}$ & $\alpha_{i n c, 1}$ & $\alpha_{d e c, 1}$ & $\alpha_{i n c, 2}$ & $\alpha_{d e c, 2}$ & & & & & \\
\hline Klinokinesis & $1 n$ & (1) & Run length & $1(0.18,1)$ & $1(0.30,1)$ & $0.05(0.020,0.094)$ & $1(0.46,1)$ & $0.3(0.18,1.01)$ & - & $500(253,1000)$ & - & 19 \\
\hline Klinokinesis & $1 t$ & (1) & Run length & $1(0.31,1)$ & $1(0.63,1)$ & $0.5(<0.094,1)$ & $0.3(0.028,0.54)$ & $0.7(0.22,1.7)$ & $0.5(0.22,7.0)$ & $1000(556,1000)$ & $10^{-3}\left(10^{-5}, 10^{-1}\right)^{*}$ & 31 \\
\hline Klinotaxis & $2 n$ & 1 & Speed & $1(0.69,1)$ & $1(0.032,1)$ & $0.6(0.047,0.96)$ & $0.02(0,0.45)$ & $0.5(0.16,3.8)$ & $0(0,0.03)$ & $600(67,1000)$ & - & 44 \\
\hline Klinotaxis & $2 t$ & 1 & Speed & $1(0.55,1)$ & $1(0.065,1)$ & $0.4(0.066,0.99)$ & $0.04(0,0.82)$ & $1(0.38,4.9)$ & $0(0,0.043)$ & - & - & 40 \\
\hline Klinotaxis & $3 n$ & 1 & Rotation & $1(0.046,1)$ & $0.05(0.0048,0.18)$ & $1(0.55,1)$ & $1(0.12,1)$ & $0.35(0.14,1.1)$ & $0.01(0.0013,0.031)$ & l) $160(64,307)$ & $1.7(1.010,1.9996) * *$ & 25 \\
\hline Klinotaxis & $3 t$ & 1 & Speed & $1(0.55,1)$ & $1(0.065,1)$ & $0.4(0.066,0.99)$ & $0.04(0,0.81)$ & $1(0.38,4.9)$ & 0 & - & $0^{* *}$ & 40 \\
\hline Tropotaxis & $4 n$ & 2 & Rotation & $1(0.25,1)$ & $1(0.58,1)$ & - & - & $1(0.18,2.8)$ & $4(1.1,10)$ & $0.8(0.59,1.1)$ & $0.5(0.25,0.75)^{* * *}$ & 18 \\
\hline Tropotaxis & $4 t$ & 2 & Rotation & $1(0.36,1)$ & $0.3(0.006,1)$ & - & - & $1(0.28,2.6)$ & $0.3(0.11,0.79)$ & - & $0.5(0.25,0.85)^{* * *}$ & 25 \\
\hline Tropotaxis & $5 n 3$ & 3 & Direction & $1(0.32,1)$ & $1(0.21,1)$ & - & - & $100(13,1000)$ & - & $0.9(<-50,1.3)$ & - & 30 \\
\hline Tropotaxis & $5 t 3$ & 3 & Direction & $0.2(0.036,1)$ & $1(0.12,1)$ & - & - & $1(0.11,4.5)$ & - & - & - & 37 \\
\hline Tropotaxis & $5 n 12$ & 12 & Direction & $1(0.27,1)$ & $1(0.074,1)$ & - & - & $10(0.16,1000)$ & - & $0.1(<-50,0.80)$ & - & 51 \\
\hline Tropotaxis & $5 t 12$ & 12 & Direction & $0.3(0.036,1)$ & $1(0.080,1)$ & - & - & $1(0.11,7.0)$ & - & - & - & 45 \\
\hline
\end{tabular}

Constraints: common noise $=0.01$, costs $=0.009,0.001$; searcher radius $=0.5$. Performance measured at a gradient decay length of 100 units or 100 searcher diameters. Maximum possible performance is 100 . Parameter values in parenthesis are approximate limits for performance within 0.5 of maximum for the model. Adaptation rates limited to [0, 1$]$. Gain limited to [-1000, 1000]. ${ }^{*} P_{\mathrm{S}}$ is the probability of changing direction.

${ }^{* *} P_{\mathrm{S}}$ is the period of oscillation. For $3 n$, performance goes to zero as $p$ approaches the integer values 1 or 2 .

$* * * P_{\mathrm{S}}$ is the separation of receptors, in fractions of a rotation. 


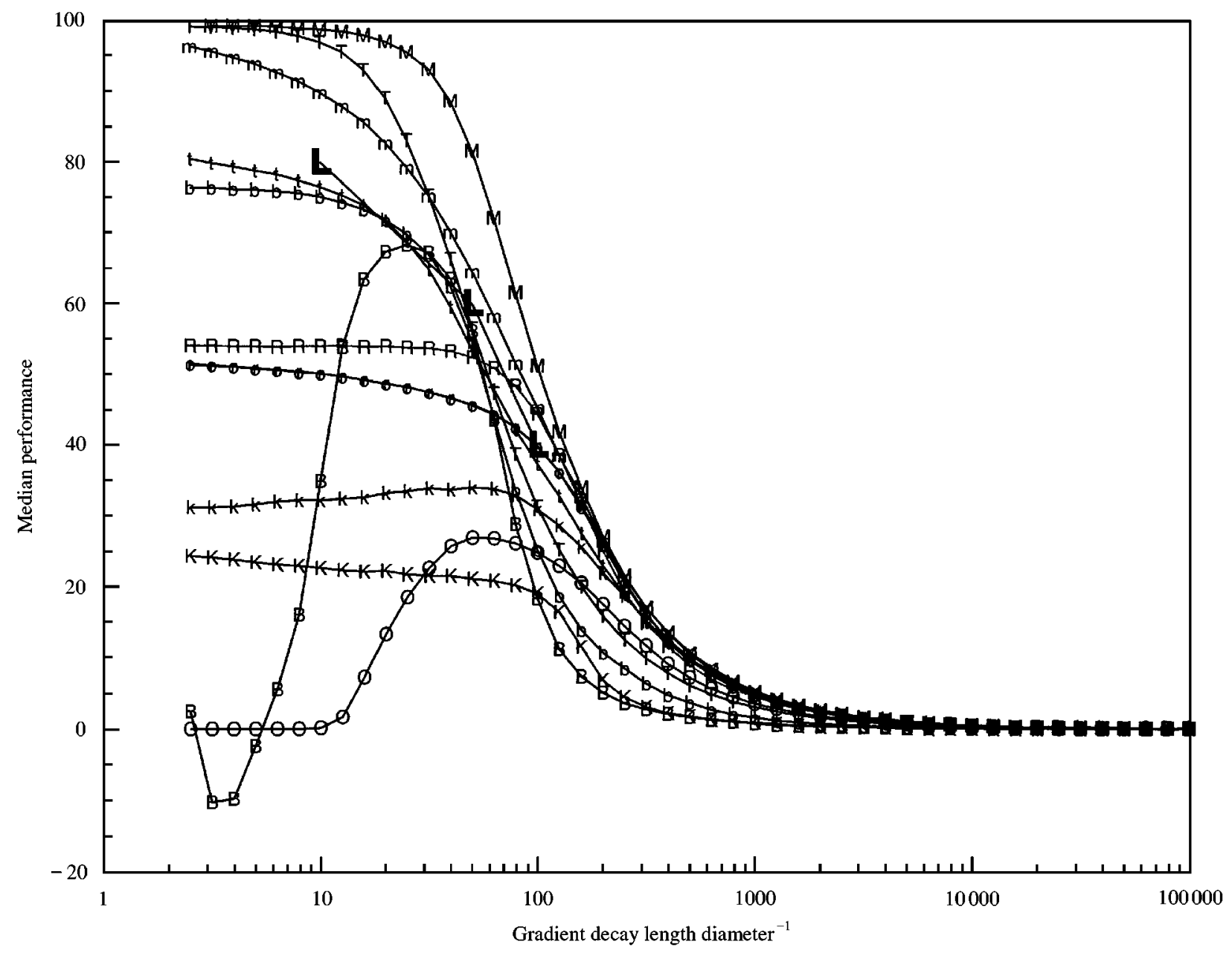

FIG. 2. Performance of strategies under parameter set 2 and data for leukocyte chemotaxis. Gradient steepness declines to the right. $S / N=1$ at a gradient decay length of 100 searcher diameters. Leukocyte data "L" is from Zigmond (1977). All other symbols represent the median performance of 10000 simulated searchers: $1 \mathrm{n}, \stackrel{\mathrm{K}-}{-} ; \mathrm{t}, \ldots \mathrm{k}-; 2 \mathrm{n},-\mathrm{R}-; 2 \mathrm{t},-\mathrm{r}-; 3 \mathrm{n},-\mathrm{O}-$;

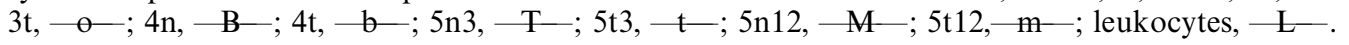

leukocyte data now fall very close to the model of tropotaxis with three receptors and threshold response, with other models somewhat more sensitive and some less sensitive. This suggests that this noise level is more realistic. In addition to greater sensitivity to shallow gradients, the lower noise level allows higher performance levels. Maximum performance for the different models ranged from 24 to over $99 \%$ of maximum. Interestingly, for a range of steep gradients, bilateral tropotaxis with continuous input-output relations perversely tended to go down the gradient significantly.

Again, in parameter set 2, klinotaxis models $2 \mathrm{n}, 2 \mathrm{t}$, and $3 \mathrm{t}$ were found to perform best with little commanded rotation. Apparently, they rely on motor noise and turning bias to provide sufficient rotation. In this situation models $2 t$ and $3 t$ are identical.

\section{Discussion}

These models do not include any elements specific to a particular size scale, and they are potentially applicable to all organisms that move over a substrate. However, for chemical stimuli, it is now understood that smooth gradients of the kinds assumed here do not exist in natural environments at large scales (say $>1 \mathrm{~cm}$ ) on the timescales of interest to the most active animals (s). Thus, these results will be most directly applicable to small organisms. However, the general features of these results may also be useful for understanding effective strategies for dealing with 
the discontinuous distributions of chemical stimuli found at larger scales. This extension is currently being explored.

Perhaps the most surprising result of these simulations is that tropotaxis with translation in any direction appears to be such a distinctly superior strategy. Although it employs more receptors, no memory is required. The two models with the best threshold response are the two models of tropotaxis with translation in any direction and the most receptors (5n12 and 5t12), and the four models with the best performance in steep gradients are the four models of tropotaxis. In addition, these models are the most robust in that they have the fewest parameters to optimize and perform well over the widest range of gradients. This suggests that amoebiod cells have the capabilities to respond very effectively to gradients. It should also raise questions about whether echinoderms like sea stars (that can move in any direction and may move so slowly that they can average concentrations long enough to see smooth gradients even in turbulent waters) are especially efficient at finding the sources of chemical stimuli.

No clear patterns were found indicating tradeoffs between good performance in shallow gradients and in steep gradients, or a general superiority of continuous or discontinuous relations between inputs and outputs.

From the formalism presented here, at least a dozen parameters emerge as important in comparing strategies for following stimulus gradients: noise in the objective intensity impinging on the organism; noise in receptors, memories, and motor outputs, including both variations with time in the function of particular components and variations between nominally identical components; costs of construction and operation of the components, including receptors, memories, translational and rotational locomotion; and the cost of time.

What are appropriate noise levels? There is very little information available on this topic. Several researchers have calculated effects of chemical fluctuations on leukocyte chemotaxis (DeLisi, 1982; Tranquillo et al., 1988), and a standard deviation of $2 \%$ has been given for the relevant fluctuations (Tranquillo et al., 1988). Levin et al. (1998) found that a standard deviation of protein copy number of $10 \%$ of the mean fit their data on bacterial swimming behavior, and it was initially guessed from lab experience that this was a good general estimate. However, the comparison in Fig. 1 with the data from leukocytes suggest that this level is generally too high. Reducing all noise levels to a standard deviation of $1 \%$ (Table 3 ) produced good agreement between the leukocyte data and the tropotaxis models, which are thought to be most appropriate (Fig. 2). The fit was particularly good for the models with a threshold.

What are appropriate costs? Little information is presently available, but some researchers are beginning to assign values to costs of information processing in animals (Laughlin et al., 1998).

How robust are the patterns observed here? The sensitivity of performance to the parameter values was evaluated by varying one parameter at a time, and determining the range of values within which performance was at least $50 \%$ of performance with the standard values, that is the peak width at half-height (Table 5). About 95\% of the limits are a factor of 2 or more from the standard value, and about $30 \%$ are an order of magnitude or more from the standard value. The ranges were also estimated using a maximum of 100 time steps in place of the usual 300 steps, and there was no apparent change in any of the parameters. Thus, most of these models are quite robust.

Comparing Figs 1 and 2, it is seen that plausible changes in noise and costs can produce significant differences in the relative performance of different models. Nonetheless, the general patterns (such as the superiority of tropotaxis with translation in any direction) remained in both cases. Clearly, the analysis could be greatly improved by obtaining more reliable values for noise and costs. In fact, a major goal of this exercise was to identify the kinds of information that are required for understanding why organisms use particular mechanisms for following stimulus gradients. Hopefully, this will inspire others to gather this kind of data.

This work was supported by a joint DARPA-ONR program through ONR grant N00014-98-1-0076. I thank Troy Keller for helpful comments on the manuscript. 


\section{REFERENCES}

Benhamou, S. \& Bovet, P. (1989). How animals use their environment: a new look at kinesis. Anim. Behav. 38, 375-383.

BerG, H. C. (1985). Physics of bacterial chemotaxis. In: Sensory Perception and Transduction in Aneural Organisms (Colombetti, G., Lenci, F. \& Song, P.-S., eds), pp. 19-30. New York: Plenum Press.

BerG, H. C. \& Tedesco, P. M. (1975). Transient response to chemotactic stimuli in Escherichia coli. Proc. Natl Acad. Sci. U.S.A. 72, 3235-3239.

BornBUSCH, A. H. (1984). Turning field size and its effects upon computer-simulated klinotactic orientation. J. theor. Biol. 107, 151-163.

Bornbusch, A. H. \& ConNer, W. E. (1986). Effects of self-steered turn size and turn bias upon simulated chemoklinotactic behavior. J. theor. Biol. 122, 7-18.

BurR, A. H. (1984). Photomovement behavior in simple invertebrates. In: Photoreception and Vision in Invertebrates (Ali, M. A., ed.). New York: Plenum Press.

CARlile, M. J. (1980). Positioning mechanisms - the role of motility, taxis and tropism in the life of microorganisms. In: Contemporary Microbial Ecology (Ellwood, D. C., Latham, M. J., Hedger, J. N., Lynch, J. M. \& Slater, J. H., eds), pp. 55-74. London: Academic Press.

DELISI, C. (1982). A theory of measurement error and its implications for spatial and temporal gradient sensing during chemotaxis. Cell Biophys. 4, 211-229.

DOUCET, P. G. \& Drost, N. J. (1985). Theoretical studies on animal orientation. II. Directional displacement in kineses. J. theor. Biol. 117, 337-361.

DunN, G. (1990). Conceptual problems with kinesis and taxis. In: Biology of the Chemotactic Response (Armitage, J. P. \& Lackie, J. M., eds), pp. 1-13. Cambridge: Cambridge University Press.

DUSENBERY, D. B. (1987). Theoretical range over which bacteria and nematodes locate plant roots using carbon dioxide. J. Chem. Ecol. 13, 1617-1624.

DUSENBERY, D. B. (1989a). Efficiency and the role of adaptation in klinokinesis. J. theor. Biol. 136, 281-293.

DUSENBERY, D. B. (1989b). The value of asymmetric signal processing in klinokinesis. Biol. Cybern. 61, 401-404.

Dusenbery, D. B. (1992). Sensory Ecology. New York: W. H. Freeman and Company.

Dusenbery, D. B. (1996). Life at Small Scale. New York: Scientific American Library.

EwER, D. W. \& BURSELl, E. (1950). A note on the classification of elementary behaviour patterns. Behavior 3, 40-47.

Ferrée, T. C. \& Lockery, S. R. (1999). Computational rules for chemotaxis in the nematode C. elegans. J. Comp. Neurosci. 6, 263-277.

Foster, K. W. \& SMYTH, R. D. (1980). Light antennas in phototactic algae. Microbiol. Rev. 44, 572-630.

Fraenkel, G. S. \& GunN, D. L. (1961). The Orientation of Animals. New York: Dover Publications, Inc.

GunN, D. L., Kennedy, J. S. \& Pielou, D. P. (1937). Classification of taxes and kineses. Nature 140, 1064.

JANDER, R. (1975). Ecological aspects of spatial orientation. Ann. Rev. Ecol. Syst. 6, 171-188.

Keller, H. U., Wilkinson, P. C., Abercrombie, M., Becker, E. L., Hirsch, J. G., Miller, M. E., Ramsay,
W. S. \& Zigmond, S. H. (1977). A proposal for the definition of terms related to locomotion of leucocytes and other cells. Clin. Exptl. Immunol. 27, 377-380.

LAUFFENBURGER, D. A. (1982). Influence of external concentration fluctuations on leukocyte chemotactic orientation. Cell Biophys. 4, 177-209.

Laughlin, S. B., VAN STEVEninck, R. R. d. R. \& ANDERSON, J. C. (1998). The metabolic cost of neural information. Nature Neurosci. 1, 36-41.

Levin, M. D., Morton-Firth, C. J., Abouhamad, W. N., BOURRET, R. B. \& BRAY, D. (1998). Origins of individual swimming behavior in bacteria. Biophys. J. 74, 175-181.

MAnN, K. H. \& LAZIER, J. R. N. (1991). Dynamics of Marine Ecosystems. Boston: Blackwell Scientific Publications.

MoOre, P. A. \& LePPER, D. M. E. (1997). Role of chemical signals in the orientation behavior of the sea star Asterias forbesi. Biol. Bull. 192, 410-417.

Morse, T. M., Ferrée, T. C. \& Lockery, S. R. (1998). Robust spatial navigation in a robot inspired by chemotaxis in Caenorhabditis elegans. Adap. Behav. 6, 393-410.

Peters, R. H. (1983). The Ecological Implications of Body Size. Cambridge: Cambridge University Press.

Pierce-Shimomura, J. T., Morse, T. M. \& Lockery, S. R. (1999). The fundamental role of pirouettes in Caenorhabditis elegans chemotaxis. J. Neurosci. 19, 9557-9569.

ROHLF, F. J. \& DAVENPORT, D. (1969). Simulation of simple models of animal behavior with a digital computer. J. theor. Biol. 23, 400-424.

Schnitzer, M. J., Block, S. M., BerG, H. C. \& Purcell, E. M. (1990). Strategies for chemotaxis. In: Biology of the Chemotactic Response (Armitage, J. P. \& Lackie, J. M., eds), pp. 15-34. Cambridge: Cambridge University Press. SCHÖNE, H. (1984). Spatial Orientation. Princeton, NJ: Princeton University Press.

Segall, J. E. (1990). Mutational studies of amoeboid chemotaxis using Dictyostelium discoideum. In: Biology of the Chemotactic Response (Armitage, J. P. \& Lackie, J. M., eds), pp. 241-272. Cambridge: Cambridge University Press.

TRANQUILLO, R. T. (1990). Theories and models of gradient perception. In: Biology of the Chemotactic Response (Armitage, J. P. \& Lackie, J. M., eds), pp. 35-75. Cambridge: Cambridge University Press.

Tranquillo, R. T., LAuffenburger, D. A. \& Zigmond, S. H. (1988). A stochastic model of leukocyte random motility and chemotaxis based on receptor binding fluctuations. J. Cell Biol. 106, 303-309.

VAN Houten, J. (1978). Two mechanisms of chemotaxis in Paramecium. J. Comp. Physiol. A 127, 167-174.

VAN Houten, J. \& VAN Houten, J. (1982). Computer simulation of Paramecium chemokinesis behavior. J. theor. Biol. 98, 453-468.

WARD, S. (1973). Chemotaxis by the nematode Caenorhabditis elegans: identification of attractants and analysis of the response by use of mutants. Proc. Natl Acad.Sci.U.S.A. 70, 817-821.

Withers, P. C. (1992). Comparative Animal Physiology. Fort Worth: Saunders College Publishing.

ZIGMOND, S. H. (1977). Ability of polymorphonuclear leukocytes to orient in gradients of chemotactic factors. J. Cell. Biol. 75, 606-616. 